\title{
Existence of solution for Hilfer fractional differential problem with nonlocal boundary condition in Banach spaces
}

\author{
Hanan A. Wahash, Mohammed S. Abdo, \\ Satish K. Panchal and Sandeep P. Bhairat
}

\begin{abstract}
This paper is devoted to study the existence of a solution to Hilfer fractional differential equation with nonlocal boundary condition in Banach spaces. We use the equivalent integral equation to study the considered Hilfer differential problem with nonlocal boundary condition. The Mönch type fixed point theorem and the measure of the noncompactness technique are the main tools in this study. We demonstrate the existence of a solution with a suitable illustrative example.
\end{abstract}

Mathematics Subject Classification (2010): 34A08, 26A33, 34A12, 34A40.

Keywords: Fractional differential equations, Hilfer fractional derivatives, existence, fixed point theorem.

\section{Introduction}

The calculus of arbitrary order has been extensively studied in the last four decades. It has been proved to be an adequate tool in almost all branches of science and engineering. Because of its widespread applications, fractional calculus is becoming an integral part of applied mathematics research. Indeed, fractional differential equations have been found useful to describe abundant phenomena in physics and engineering, and the modest amount of work in this direction has taken place, see [1, 4, 9] and references therein. For basic development and theoretical applications of fractional differential equations, see $[15,17]$.

In the past two decades, the fractional differential equations are extensively studied for existence, uniqueness, continuous dependence and stability of the solution. For some fundamental results in existence theory of various fractional differential problems with initial and boundary conditions, see survey papers [1, 4], the monograph [17], the research papers $[2,3,7,5,6,8,9,10,11,12,16,20,22]$ and references therein. 
Recently, in [22], Wang and Zhang obtained some existence of the solutions of IVP for the class of Hilfer FDEs:

$$
\begin{aligned}
& D_{0^{+}}^{\mu, \nu} z(t)=f(t, z(t)), \quad 0<\mu<1,0 \leq \nu \leq 1, t \in(a, b] \\
& I_{a^{+}}^{1-\gamma} z\left(a^{+}\right)=\sum_{k=1}^{m} \lambda_{k} z\left(\tau_{k}\right), \tau_{k} \in(a, b], \mu \leq \gamma=\mu+\nu(1-\mu),
\end{aligned}
$$

by using fixed point theorems of Krasnoselskii and Schauder.

In the year 2018, Thabet et al. [19] investigated the existence of a solution to BVP for Hilfer FDEs:

$$
\begin{aligned}
& D_{a^{+}}^{\mu, \nu} z(t)=f(t, z(t), S z(t)), 0<\mu<1,0 \leq \nu \leq 1, \quad t \in(a, b], \\
& I_{a^{+}}^{1-\gamma}\left[u z\left(a^{+}\right)+v z\left(b^{-}\right)\right]=w, \quad \mu \leq \gamma=\mu+\nu(1-\mu), u, v, w \in \mathbb{R},
\end{aligned}
$$

by using the Mönch fixed point theorem.

Motivated by works cited above, in this paper, we consider the nonlocal boundary value problem for a class of Hilfer fractional differential equations (HNBVP):

$$
\begin{gathered}
D_{a^{+}}^{\mu, \nu} z(t)=f(t, z(t)), \quad 0<\mu<1,0 \leq \nu \leq 1, t \in(a, b], \\
I_{a^{+}}^{1-\gamma} c z\left(a^{+}\right)+I_{a^{+}}^{1-\gamma} d z\left(b^{-}\right)=\sum_{k=1}^{m} \lambda_{k} z\left(\tau_{k}\right), \tau_{k} \in(a, b], \mu \leq \gamma=\mu+\nu(1-\mu),
\end{gathered}
$$

where $D_{a^{+}}^{\mu, \nu}$ is the Hilfer fractional derivative of order $\mu$ and type $\nu, I_{a^{+}}^{1-\gamma}$ is the Riemann-Liouville fractional integral of order $1-\gamma, f:(a, b] \times E \rightarrow E$ be a function such that $f(t, z) \in C_{1-\gamma}([a, b], E)$ for any $z \in C_{1-\gamma}([a, b], E), E$ is a Banach space, $c, d \in \mathbb{R}$, and $\tau_{k}(k=1,2, \ldots, m)$ are prefixed points satisfying $a<\tau_{1}<\tau_{2}<\ldots<$ $\tau_{m}<b, \lambda_{k}$ are real numbers.

The measure of noncompactness technique and a fixed point theorem of Monch type are the main tools in this analysis.

The paper is organized as follows: Some preliminary concepts related to our problem are listed in Section 2 which will be useful in the sequel. In Section 3, we first establish an equivalent integral equation of BVP and then we present the existence of its solution. An illustrative example is provided in the last section.

\section{Preliminaries}

In this section, we present some definitions, lemmas and weighted spaces which are useful in further development of this paper.

Let $J_{1}=[a, b]$ and $J_{2}=(a, b](\infty<a<b<+\infty)$. Let $C\left(J_{1}, E\right)$, be the Banach spaces of all continuous function $g: J_{1} \rightarrow E$ with the norm $\|g\|_{\infty}=\sup \left\{|g(t)| ; t \in J_{1}\right\}$. Here $L^{p}\left(J_{1}, E\right), p>1$, is the Banach space of measurable functions on $J_{1}$ with the $L^{p}$ norm where

$$
\|g\|_{L^{p}}=\left(\int_{a}^{b}|g(s)|^{p} d s\right)^{\frac{1}{p}}<\infty .
$$


Let $L^{\infty}\left(J_{1}, E\right)$ be the Banach space of measurable functions $z: J_{1} \longrightarrow E$ which are bounded and equipped with the norm $\|z\|_{L^{\infty}}=\inf \left\{e>0:\|z\| \leq e\right.$, a.e $\left.t \in J_{1}\right\}$. Moreover, for a given set $\mathcal{V}$ of functions $v: J_{1} \longrightarrow E$ let us denote by

$$
\begin{gathered}
\mathcal{V}(t)=\left\{v(t): v \in \mathcal{V} ; t \in J_{1}\right\}, \\
\mathcal{V}\left(J_{1}\right)=\left\{v(t): v \in \mathcal{V} ; t \in J_{1}\right\} .
\end{gathered}
$$

Definition 2.1. [17] Let $\mu>0$. The left sided Riemann-Liouville fractional integral of order $\mu$ of $g \in L^{1}\left(J_{1}, E\right)$ is defined by

$$
I_{a^{+}}^{\mu} g(t)=\frac{1}{\Gamma(\mu)} \int_{a}^{t}(t-s)^{\mu-1} g(s) d s, \quad t>a,
$$

where $\Gamma(\cdot)$ is the Euler's Gamma function and $a \in \mathbb{R}$.

Definition 2.2. [17] Let $n-1<\mu<n$. The left sided Riemann-Liouville and Caputo fractional derivatives of order $\mu$ of $g \in L^{1}\left(J_{1}, E\right)$ are defined by

$$
D_{a^{+}}^{\mu} g(t)=\frac{1}{\Gamma(n-\mu)} \frac{d^{n}}{d t^{n}} \int_{a}^{t}(t-s)^{n-\mu-1} g(s) d s, t>a,
$$

and

$$
{ }^{C} D_{a^{+}}^{\mu} g(t)=\frac{1}{\Gamma(n-\mu)} \int_{a}^{t}(t-s)^{n-\mu-1} g^{(n)}(s) d s, t>a,
$$

respectively, where $n=[\mu]+1$, and $[\mu]$ denotes the integer part of $\mu$.

Definition 2.3. [15] The left sided Hilfer fractional derivative of function $g \in L^{1}\left(J_{1}, E\right)$ of order $0<\mu<1$ and type $0 \leq \nu \leq 1$ is denoted as $D_{a^{+}}^{\mu, \nu}$ and defined by

$$
D_{a^{+}}^{\mu, \nu} g(t)=I_{a^{+}}^{\nu(1-\mu)} D I_{a^{+}}^{(1-\nu)(1-\mu)} g(t), D=\frac{d}{d t} .
$$

where $I_{a^{+}}^{\mu}$ and $D_{a^{+}}^{\mu}$ are Riemann-Liouville fractional integral and derivative defined by (2.1) and (2.2), respectively.

Remark 2.4. From Definition 2.3, we observe that:

(i) The operator $D_{a^{+}}^{\mu, \nu}$ can be written as

$$
D_{a^{+}}^{\mu, \nu}=I_{a^{+}}^{\nu(1-\mu)} D I_{a^{+}}^{(1-\gamma)}=I_{a^{+}}^{\nu(1-\mu)} D^{\gamma}, \gamma=\mu+\nu(1-\mu) .
$$

(ii) The Hilfer fractional derivative can be regarded as an interpolator between the Riemann-Liouville derivative $(\nu=0)$ and Caputo derivative $(\nu=1)$ as

$$
D_{a^{+}}^{\mu, \nu}=\left\{\begin{array}{l}
D I_{a^{+}}^{(1-\mu)}=D_{a^{+}}^{\mu}, \text { if } \nu=0 \\
I_{a^{+}}^{(1-\mu)} D={ }^{C} D_{a^{+}}^{\mu}, \text { if } \nu=1 .
\end{array}\right.
$$

(iii) In particular, if $\gamma=\mu+\nu(1-\mu)$, then

$$
\left(D_{a^{+}}^{\mu, \nu} g\right)(t)=\left(I_{a^{+}}^{\nu(1-\mu)}\left(D_{a^{+}}^{\gamma} g\right)\right)(t)
$$

where $\left(D_{a^{+}}^{\gamma} g\right)(t)=\frac{d}{d t}\left(I_{a^{+}}^{(1-\nu)(1-\mu)} g\right)(t)$. 
Definition 2.5. [17] Let $0 \leq \gamma<1$. The weighted spaces $C_{\gamma}\left(J_{1}, E\right)$ and $C_{1-\gamma}^{n}\left(J_{1}, E\right)$ are defined by

$$
C_{\gamma}\left(J_{1}, E\right)=\left\{g: J_{2} \rightarrow E:(t-a)^{\gamma} g(t) \in C\left(J_{1}, E\right)\right\},
$$

and

$$
C_{\gamma}^{n}\left(J_{1}, E\right)=\left\{g: J_{2} \rightarrow E, g \in C^{n-1}\left(J_{1}, E\right): g^{(n)}(t) \in C_{\gamma}\left(J_{1}, E\right)\right\}, n \in \mathbb{N}
$$

with the norms

$$
\|g\|_{C_{\gamma}}=\left\|(t-a)^{\gamma} g\right\|_{C}=\sup \left\{\left|(t-a)^{\gamma} g(t)\right|: t \in J_{1}\right\}
$$

and

$$
\|g\|_{C_{1-\gamma}^{n}}=\sum_{k=0}^{n-1}\left\|g^{(k)}\right\|_{C}+\left\|g^{(n)}\right\|_{C_{1-\gamma}},
$$

respectively. Furthermore we recall following weighted spaces

$$
C_{1-\gamma}^{\mu, \nu}\left(J_{1}, E\right)=\left\{g \in C_{1-\gamma}\left(J_{1}, E\right): D_{a^{+}}^{\mu, \nu} g \in C_{1-\gamma}\left(J_{1}, E\right)\right\}, \quad \gamma=\mu+\nu(1-\mu)
$$

and

$$
C_{1-\gamma}^{\gamma}\left(J_{1}, E\right)=\left\{g \in C_{1-\gamma}\left(J_{1}, E\right): D_{a^{+}}^{\gamma} g \in C_{1-\gamma}\left(J_{1}, E\right)\right\}, \quad \gamma=\mu+\nu(1-\mu) .
$$

Let $0<\mu<1,0 \leq \nu \leq 1$ and $\gamma=\mu+\nu(1-\mu)$. Clearly, $D_{a^{+}}^{\mu, \nu} g=I_{a^{+}}^{\nu(1-\mu)} D_{a^{+}}^{\gamma} g$ and $C_{1-\gamma}^{\gamma}\left(J_{1}, E\right) \subset C_{1-\gamma}^{\mu, \nu}\left(J_{1}, E\right)$.

Lemma 2.6. [9] If $\mu>0, \nu>0$ and $g \in L^{1}\left(J_{1}, E\right)$ for $t \in[a, b]$, then the following properties hold:

$$
\left(I_{a^{+}}^{\mu} I_{a^{+}}^{\nu} g\right)(t)=\left(I_{a^{+}}^{\mu+\nu} g\right)(t) \text { and }\left(D_{a^{+}}^{\mu} I_{a^{+}}^{\nu} g\right)(t)=g(t) .
$$

In particular, if $g \in C_{\gamma}\left(J_{1}, E\right)$ or $g \in C\left(J_{1}, E\right)$, then the above properties hold for each $t \in J_{2}$ or $t \in J_{1}$ respectively.

Lemma 2.7. [17] Let $\mu>0$ and $\delta>0$. Then for $t>a$, we have

(i). $I_{a^{+}}^{\mu}(t-a)^{\delta-1}=\frac{\Gamma(\delta)}{\Gamma(\delta+\mu)}(t-a)^{\delta+\mu-1}$,

(ii). $D_{a^{+}}^{\mu}(t-a)^{\mu-1}=0, \quad \mu \in(0,1)$.

Lemma 2.8. [15] Let $\mu>0, \nu>0$ and $\gamma=\mu+\nu(1-\mu)$. If $g \in C_{1-\gamma}^{\gamma}\left(J_{1}, E\right)$, then

$$
I_{a^{+}}^{\gamma} D_{a^{+}}^{\gamma} g=I_{a^{+}}^{\mu} D_{a^{+}}^{\mu, \nu} g, D_{a^{+}}^{\gamma} I_{a^{+}}^{\mu} g=D_{a^{+}}^{\nu(1-\mu)} g .
$$

Lemma 2.9. [15] Let $0<\mu<1,0 \leq \nu \leq 1$ and $g \in C_{1-\gamma}\left(J_{1}, E\right)$. Then

$$
I_{a^{+}}^{\mu} D_{a^{+}}^{\mu, \nu} g(t)=g(t)-\frac{I_{a^{+}}^{(1-\nu)(1-\mu)} g(a)}{\Gamma(\mu+\nu(1-\mu))}(t-a)^{\mu+\nu(1-\mu)-1} .
$$

Moreover, if $\gamma=\mu+\nu(1-\mu), g \in C_{1-\gamma}\left(J_{1}, E\right)$ and $I_{a^{+}}^{1-\gamma} g \in C_{1-\gamma}^{1}\left(J_{1}, E\right)$, then

$$
I_{a^{+}}^{\gamma} D_{a^{+}}^{\gamma} g(t)=g(t)-\frac{I_{a^{+}}^{1-\gamma} g(a)}{\Gamma(\gamma)}(t-a)^{\gamma-1} .
$$


Lemma 2.10. [16] If $0<\mu \leq \gamma<1$ and $g \in C_{\gamma}\left(J_{1}, E\right)$, then

$$
\left(I_{a^{+}}^{\mu} g\right)(a)=\lim _{t \rightarrow a^{+}} I_{a^{+}}^{\mu} g(t)=0 .
$$

Lemma 2.11. [18] Let $E$ be a Banach space and let $\Upsilon_{E}$ be the bounded subsets of $E$. The Kuratowski measure of noncompactness is the map $\alpha: \Upsilon_{E} \longrightarrow[0, \infty)$ defined by

$$
\alpha(\mathcal{S})=\inf \left\{\varepsilon>0: \mathcal{S} \subset \cup_{i=1}^{m} \mathcal{S}_{i} \text { and the } \operatorname{diam}\left(\mathcal{S}_{i}\right) \leq \varepsilon\right\} ; \mathcal{S} \subset \Upsilon_{E}
$$

Lemma 2.12. [14, 13] For all nonempty subsets $\mathcal{S}_{1}, \mathcal{S}_{2} \subset$ E. The Kuratowski measure of noncompactness $\alpha(\cdot)$ satisfies the following properties:

1. $\alpha(\mathcal{S})=0 \Longleftrightarrow \overline{\mathcal{S}}$ is compact ( $\mathcal{S}$ is relatively compact);

2. $\alpha(\mathcal{S})=\alpha(\overline{\mathcal{S}})=\alpha(\operatorname{conv} \mathcal{S})$, where where $\overline{\mathcal{S}}$ and conv $\mathcal{S}$ denote the closure and convex hull of the bounded set $\mathcal{S}$ respectively;

3. $\mathcal{S}_{1} \subset \mathcal{S}_{2} \Longrightarrow \alpha\left(\mathcal{S}_{1}\right) \leq \alpha\left(\mathcal{S}_{2}\right)$;

4. $\alpha\left(\mathcal{S}_{1}+\mathcal{S}_{2}\right) \leq \alpha\left(\mathcal{S}_{1}\right)+\alpha\left(\mathcal{S}_{2}\right)$, where $\mathcal{S}_{1}+\mathcal{S}_{2}=\left\{s_{1}+s_{2}: s \in \mathcal{S}_{1}, s \in \mathcal{S}_{2}\right\}$;

5. $\alpha(\kappa \mathcal{S})=|\kappa| \alpha(\overline{\mathcal{S}}), \kappa \in \mathbb{R}$;

Lemma 2.13. [18] Let $\mathbb{B}$ be a bounded, closed and convex subset of a Banach space $E$ such that $0 \in \mathbb{B}$, and let $\mathcal{T}$ be a continuous mapping of $\mathbb{B}$ into itself. If for every subset $\mathcal{V}$ of $\mathbb{B}$

$$
\mathcal{V}=\overline{c o} \mathcal{T}(\mathcal{V}) \text { or } \mathcal{V}=\mathcal{T}(\mathcal{V}) \cup\{0\} \Longrightarrow \alpha(\mathcal{V})=0
$$

holds. Then $\mathcal{T}$ has a fixed point.

Lemma 2.14. [21] Let $\mathbb{B}$ be a bounded, closed and convex subset of a Banach space $C\left(J_{1}, E\right), F$ is a continuous function on $J_{1} \times J_{1}$; and a function $f: J_{1} \times E \longrightarrow E$ satisfying the Carathéodory conditions, and assume there exists $\rho \in L^{P}\left(J_{1}, \mathbb{R}^{+}\right)$such that, for each $t \in J_{1}$ and each bounded set $\mathbb{B}^{*} \subset E$; one has

$$
\lim _{r \longrightarrow 0^{+}} \alpha\left(f\left(J_{t, r} \times \mathbb{B}^{*}\right)\right) \leq \rho(t) \alpha\left(\mathbb{B}^{*}\right) \text {, where } J_{t, r} \in[t-r, t] \cap J_{1} .
$$

If $\mathcal{V}$ is an equicontinuous subset of $\mathbb{B}$; then

$$
\alpha\left(\left\{\int_{J_{1}} F(t, s) f(s, z(s)) d s: z \in \mathcal{V}\right\}\right) \leq \int_{J_{1}}\|F(t, s)\| \rho(s) \alpha(\mathcal{V}(s)) d s .
$$

Lemma 2.15. [10] Let $\gamma=\mu+\nu(1-\mu)$ where $0<\mu<1$ and $0 \leq \nu \leq 1$. Let $f:(a, b] \times \mathbb{R} \rightarrow \mathbb{R}$ be a function such that $f(t, z) \in C_{1-\gamma}[a, b]$ for any $z \in C_{1-\gamma}[a, b]$. If $z \in C_{1-\gamma}^{\gamma}[a, b]$, then $z$ satisfies IVP

$$
\begin{gathered}
D_{a^{+}}^{\mu, \nu} z(t)=f(t, z(t)), \quad 0<\mu<1,0 \leq \nu \leq 1, t \in[a, b], \\
I_{a^{+}}^{1-\gamma} z\left(0^{+}\right)=z_{a}, \quad \mu \leq \gamma
\end{gathered}
$$

if and only if $z$ satisfies the Volterra integral equation

$$
z(t)=\frac{z_{a}}{\Gamma(\gamma)}(t-a)^{\gamma-1}+\frac{1}{\Gamma(\mu)} \int_{a}^{t}(t-s)^{\mu-1} f(s, z(s)) d s, \quad t>a .
$$




\section{Main results}

Now we prove the existence of solution of HNBVP (1.5)-(1.6) in $C_{1-\gamma}^{\gamma}\left(J_{1}, E\right) \subset$ $C_{1-\gamma}^{\mu, \nu}\left(J_{1}, E\right)$ under measure of noncompactness technique and a fixed point theorem of Mönch type.

Definition 3.1. A function $z \in C_{1-\gamma}^{\gamma}\left(J_{1}, E\right)$ is said to be a solution of HNBVP (1.5)(1.6) if $z$ satisfies the fractional differential equation $D_{a^{+}}^{\mu, \nu} z(t)=f(t, z(t))$ on $J_{2}$, and the nonlocal boundary condition $I_{a^{+}}^{1-\gamma}\left[c z\left(a^{+}\right)+d z\left(b^{-}\right)\right]=\sum_{k=1}^{m} \lambda_{k} z\left(\tau_{k}\right)$.

In the beginning, we need the following axiom lemma:

Lemma 3.2. Let $0<\mu<1,0 \leq \nu \leq 1$ where $\gamma=\mu+\nu(1-\mu)$, and $f: J_{2} \times E \rightarrow E$ be $a$ function such that $f(t, z) \in C_{1-\gamma}\left(J_{1}, E\right)$ for any $z \in C_{1-\gamma}\left(J_{1}, E\right)$. If $z \in C_{1-\gamma}^{\gamma}\left(J_{1}, E\right)$, then $z$ satisfies HNBVP (1.5)-(1.6) if and only if $z$ satisfies the following integral equation

$$
\begin{aligned}
z(t)= & \frac{(t-a)^{\gamma-1}}{\Gamma(\gamma)} \frac{1}{(c+d-A)} \sum_{k=1}^{m} \frac{\lambda_{k}}{\Gamma(\mu)} \int_{a}^{\tau_{k}}\left(\tau_{k}-s\right)^{\mu-1} f(s, z(s)) d s \\
& -\frac{(t-a)^{\gamma-1}}{\Gamma(\gamma)} \frac{d}{(c+d-A)} \frac{1}{\Gamma(1-\gamma+\mu)} \int_{a}^{b}(b-s)^{-\gamma+\mu} f(s, z(s)) d s \\
& +\frac{1}{\Gamma(\mu)} \int_{a}^{t}(t-s)^{\mu-1} f(s, z(s)) d s
\end{aligned}
$$

where $A=\sum_{k=1}^{m} \lambda_{k} \frac{\left(\tau_{k}-a\right)^{\gamma-1}}{\Gamma(\gamma)}$, and $c+d \neq A$.

Proof. In view of Lemma 2.15, the solution of (1.5) can be written as

$$
z(t)=\frac{I_{a^{+}}^{1-\gamma} z\left(a^{+}\right)}{\Gamma(\gamma)}(t-a)^{\gamma-1}+\frac{1}{\Gamma(\mu)} \int_{a}^{t}(t-s)^{\mu-1} f(s, z(s)) d s, \quad t>a .
$$

Applying $I_{a^{+}}^{1-\gamma}$ on both sides of (3.2) and taking the limit $t \rightarrow b^{-}$, we obtain

$$
I_{a^{+}}^{1-\gamma} z\left(b^{-}\right)=I_{a^{+}}^{1-\gamma} z\left(a^{+}\right)+\frac{1}{\Gamma(1-\gamma+\mu)} \int_{a}^{b}(b-s)^{-\gamma+\mu} f(s, z(s)) d s .
$$

Now, we substitute $t=\tau_{k}$ in (3.2) and multiply by $\lambda_{k}$ to obtain

$$
\lambda_{k} z\left(\tau_{k}\right)=\lambda_{k}\left[\frac{I_{a^{+}}^{1-\gamma} z\left(a^{+}\right)}{\Gamma(\gamma)}\left(\tau_{k}-a\right)^{\gamma-1}+\frac{1}{\Gamma(\mu)} \int_{a}^{\tau_{k}}\left(\tau_{k}-s\right)^{\mu-1} f(s, z(s)) d s\right]
$$

Using the nonlocal boundary condition (1.6) with (3.3) and (3.4), we have

$$
\begin{aligned}
I_{a^{+}}^{1-\gamma} z\left(a^{+}\right)= & \frac{1}{c} \sum_{k=1}^{m} \lambda_{k} z\left(\tau_{k}\right)-\frac{d}{c} I_{a^{+}}^{1-\gamma} z\left(a^{+}\right) \\
& +\frac{d}{c \Gamma(1-\gamma+\mu)} \int_{a}^{b}(b-s)^{-\gamma+\mu} f(s, z(s)) d s .
\end{aligned}
$$


Therefore, by (3.4), we have

$$
\begin{aligned}
I_{a^{+}}^{1-\gamma} z\left(a^{+}\right)= & \frac{1}{c} \sum_{k=1}^{m} \lambda_{k} \frac{I_{a^{+}}^{1-\gamma} z\left(a^{+}\right)}{\Gamma(\gamma)}\left(\tau_{k}-a\right)^{\gamma-1} \\
& +\frac{1}{c} \sum_{k=1}^{m} \frac{\lambda_{k}}{\Gamma(\mu)} \int_{a}^{\tau_{k}}\left(\tau_{k}-s\right)^{\mu-1} f(s, z(s)) d s \\
& -\frac{d}{c} I_{a^{+}}^{1-\gamma} z\left(a^{+}\right)-\frac{d}{c} \frac{1}{\Gamma(1-\gamma+\mu)} \int_{a}^{b}(b-s)^{-\gamma+\mu} f(s, z(s)) d s . \\
= & \frac{1}{(c+d-A)} \sum_{k=1}^{m} \frac{\lambda_{k}}{\Gamma(\mu)} \int_{a}^{\tau_{k}}\left(\tau_{k}-s\right)^{\mu-1} f(s, z(s)) d s \\
& -\frac{d}{(c+d-A)} \frac{1}{\Gamma(1-\gamma+\mu)} \int_{a}^{b}(b-s)^{-\gamma+\mu} f(s, z(s)) d s .
\end{aligned}
$$

Submitting (3.5) into (3.2), we obtain

$$
\begin{aligned}
z(t)= & \frac{(t-a)^{\gamma-1}}{\Gamma(\gamma)} \frac{1}{(c+d-A)} \sum_{k=1}^{m} \frac{\lambda_{k}}{\Gamma(\mu)} \int_{a}^{\tau_{k}}\left(\tau_{k}-s\right)^{\mu-1} f(s, z(s)) d s \\
& -\frac{(t-a)^{\gamma-1}}{\Gamma(\gamma)} \frac{d}{(c+d-A)} \frac{1}{\Gamma(1-\gamma+\mu)} \int_{a}^{b}(b-s)^{-\gamma+\mu} f(s, z(s)) d s \\
& +\frac{1}{\Gamma(\mu)} \int_{a}^{t}(t-s)^{\mu-1} f(s, z(s)) d s .
\end{aligned}
$$

Conversely, applying $I_{a^{+}}^{1-\gamma}$ on both sides of (3.1), then it follows from Lemmas 2.6, 2.7 , and some simple computations that

$$
\begin{aligned}
& I_{a^{+}}^{1-\gamma}\left(c z\left(a^{+}\right)+d z\left(b^{-}\right)\right) \\
= & \frac{c}{(c+d-A)} \sum_{k=1}^{m} \frac{\lambda_{k}}{\Gamma(\mu)} \int_{a}^{\tau_{k}}\left(\tau_{k}-s\right)^{\mu-1} f(s, z(s)) d s \\
& -\frac{c d}{(c+d-A)} \frac{1}{\Gamma(1-\gamma+\mu)} \int_{a}^{b}(b-s)^{-\gamma+\mu} f(s, z(s)) d s \\
& +\frac{d}{(c+d-A)} \sum_{k=1}^{m} \frac{\lambda_{k}}{\Gamma(\mu)} \int_{a}^{\tau_{k}}\left(\tau_{k}-s\right)^{\mu-1} f(s, z(s)) d s \\
& -\frac{d^{2}}{(c+d-A)} \frac{1}{\Gamma(1-\gamma+\mu)} \int_{a}^{b}(b-s)^{-\gamma+\mu} f(s, z(s)) d s \\
+ & \frac{d}{\Gamma(1-\gamma+\mu)} \int_{a}^{b}(b-s)^{-\gamma+\mu} f(s, z(s)) d s .
\end{aligned}
$$


Which implies

$$
\begin{aligned}
& I_{a^{+}}^{1-\gamma}\left(c z\left(a^{+}\right)+d z\left(b^{-}\right)\right) \\
= & \left(\frac{c}{(c+d-A)}+\frac{d}{(c+d-A)}\right) \sum_{k=1}^{m} \frac{\lambda_{k}}{\Gamma(\mu)} \int_{a}^{\tau_{k}}\left(\tau_{k}-s\right)^{\mu-1} f(s, z(s)) d s \\
& -\left(d-\frac{c d}{(c+d-A)}-\frac{d^{2}}{(c+d-A)}\right) \int_{a}^{b} \frac{(b-s)^{-\gamma+\mu}}{\Gamma(1-\gamma+\mu)} f(s, z(s)) d s \\
= & \frac{c+d}{(c+d-A)} \sum_{k=1}^{m} \frac{\lambda_{k}}{\Gamma(\mu)} \int_{a}^{\tau_{k}}\left(\tau_{k}-s\right)^{\mu-1} f(s, z(s)) d s \\
& -\frac{A d}{(c+d-A)} \frac{1}{\Gamma(1-\gamma+\mu)} \int_{a}^{b}(b-s)^{-\gamma+\mu} f(s, z(s)) d s .
\end{aligned}
$$

From (3.4) and (3.5), we conclude that

$$
I_{a^{+}}^{1-\gamma}\left(c z\left(a^{+}\right)+d z\left(b^{-}\right)\right)=\sum_{k=1}^{m} \lambda_{k} z\left(\tau_{k}\right)
$$

which shows that the nonlocal boundary condition (1.6) is satisfied.

Next, applying $D_{a^{+}}^{\gamma}$ on both sides of (3.1) and using Lemmas 2.7 and 2.8, we have

$$
D_{a^{+}}^{\gamma} z(t)=D_{a^{+}}^{\nu(1-\mu)} f(t, z(t)) .
$$

Since $z \in C_{1-\gamma}^{\gamma}\left(J_{1}, E\right)$ and by definition of $C_{1-\gamma}^{\gamma}\left(J_{1}, E\right)$, we have $D_{a^{+}}^{\gamma} z \in C_{1-\gamma}\left(J_{1}, E\right)$, therefore, $D_{a^{+}}^{\nu(1-\mu)} f=D I_{a^{+}}^{1-\nu(1-\mu)} f \in C_{1-\gamma}\left(J_{1}, E\right)$. For $f \in C_{1-\gamma}\left(J_{1}, E\right)$, it is clear that $I_{a^{+}}^{1-\nu(1-\mu)} f \in C_{1-\gamma}\left(J_{1}, E\right)$. Hence $f$ and $I_{a^{+}}^{1-\nu(1-\mu)} f$ satisfy the hypothesis of Lemma 2.9 .

Now, by applying $I_{a^{+}}^{\nu(1-\mu)}$ on both sides of (3.7), we have

$$
I_{a^{+}}^{\nu(1-\mu)} D_{a^{+}}^{\gamma} z(t)=I_{a^{+}}^{\nu(1-\mu)} D_{a^{+}}^{\nu(1-\mu)} f(t, z(t)) .
$$

Using Remark 2.4 (i), relation (3.7) and Lemma 2.9, we get

$$
D_{a^{+}}^{\mu, \nu} z(t)=f(t, z(t))-\frac{I_{a^{+}}^{1-\nu(1-\mu)} f(a, z(a))}{\Gamma(\nu(1-\mu))}(t-a)^{\nu(1-\mu)-1}, \text { for all } t \in J_{2} .
$$

By Lemma 2.10, we have $I_{a^{+}}^{1-\nu(1-\mu)} f(a, z(a))=0$. Therefore $D_{a^{+}}^{\mu, \nu} z(t)=f(t, z(t))$. This completes the proof.

To prove the existence of solutions for the problem at hand, let us make the following hypotheses.

(H1) The function $f: J_{2} \times E \rightarrow E$ satisfies the Carathèodory conditions.

(H2) $f: J_{2} \times E \rightarrow E$ is a function such that $f(\cdot, z(\cdot)) \in C_{1-\gamma}^{\nu(1-\mu)}\left(J_{1}, E\right)$ for any $z \in C_{1-\gamma}\left(J_{1}, E\right)$ and there exists $\rho \in L^{p}\left(J_{1}, \mathbb{R}^{+}\right)$with $p>\frac{1}{\mu}$ and $p>\frac{1}{\gamma}$ such that

$$
\|f(t, z)\| \leq \rho(t)\|z\|
$$

for each $t \in J_{2}$, and all $z \in E$. 
(H3) The following inequalities

$$
\begin{aligned}
\mathcal{G}: & =\left(\frac{1}{\Gamma(\gamma)} \frac{\left(\Lambda_{q, \mu, \gamma}\right)^{\frac{1}{q}}}{(c+d-A)} \sum_{k=1}^{m} \frac{\lambda_{k}}{\Gamma(\mu)}\left(\tau_{k}-a\right)^{\gamma+\mu-1}\right. \\
& \left.+\left(\frac{1}{\Gamma(\gamma)}\left|\frac{d}{(c+d-A)}\right| \frac{\left(\Delta_{q, \mu, \gamma}\right)^{\frac{1}{q}}}{\Gamma(1-\gamma+\mu)}+\frac{\left(\Lambda_{q, \mu, \gamma}\right)^{\frac{1}{q}}}{\Gamma(\mu)}\right)(b-a)^{\mu}\right)\|\rho\|_{L^{p}}<1,
\end{aligned}
$$

and

$$
\begin{aligned}
L^{*}: \quad & =\left(\frac{m}{\Gamma(\gamma)} \frac{(b-a)^{\gamma-1}}{(c+d-A)} \sum_{k=1}^{m} \frac{\lambda_{k}\left(\tau_{k}-a\right)^{\mu}}{\Gamma(\mu+1)}\right. \\
& \left.+\left(\frac{1}{\Gamma(\gamma)}\left|\frac{d}{(c+d-A)}\right| \frac{1}{\Gamma(-\gamma+\mu)}+\frac{1}{\Gamma(\mu+1)}\right)(b-a)^{\mu}\right)\|\rho\|_{L^{p}}<1
\end{aligned}
$$

hold, where $q>1, \frac{1}{p}+\frac{1}{q}=1$ and

$$
\begin{aligned}
\Lambda_{q, \mu, \gamma} & :=\frac{\Gamma(q(\mu-1)+1) \Gamma(q(\gamma-1)+1)}{\Gamma(q(\mu+\gamma-2)+2)}, \\
\Delta_{q, \mu, \gamma} & :=\frac{\Gamma(q(\mu-\gamma)+1) \Gamma(q(\gamma-1)+1)}{\Gamma(q(\mu-1)+2)} .
\end{aligned}
$$

Now, we are ready to prove the existence of solutions for the HNBVP (1.5)-(1.6), which is based on fixed point theorem of Mönch's type.

Theorem 3.3. Assume that (H1)-(H3) are satisfied. Then HNBVP (1.5)-(1.6) has at least one solution in $C_{1-\gamma}^{\gamma}\left(J_{1}, E\right) \subset C_{1-\gamma}^{\mu, \nu}\left(J_{1}, E\right)$.

Proof. Transform the problem (1.5)-(1.6) into a fixed point problem. Define the operator $\mathcal{T}: C_{1-\gamma}\left(J_{1}, E\right) \longrightarrow C_{1-\gamma}\left(J_{1}, E\right)$ as

$$
\begin{aligned}
\mathcal{T} z(t)= & z(t)=\frac{(t-a)^{\gamma-1}}{\Gamma(\gamma)} \frac{1}{(c+d-A)} \sum_{k=1}^{m} \frac{\lambda_{k}}{\Gamma(\mu)} \int_{a}^{\tau_{k}}\left(\tau_{k}-s\right)^{\mu-1} f(s, z(s)) d s \\
& -\frac{(t-a)^{\gamma-1}}{\Gamma(\gamma)} \frac{d}{(c+d-A)} \frac{1}{\Gamma(1-\gamma+\mu)} \int_{a}^{b}(b-s)^{-\gamma+\mu} f(s, z(s)) d s \\
& +\frac{1}{\Gamma(\mu)} \int_{a}^{t}(t-s)^{\mu-1} f(s, z(s)) d s .
\end{aligned}
$$

Clearly, from Lemma 3.2, the fixed points of $\mathcal{T}$ are solutions to (1.5)-(1.6). Let

$$
\mathbb{B}_{R}=\left\{z \in C_{1-\gamma}\left(J_{1}, E\right):\|z\|_{C_{1-\gamma}} \leq R\right\} .
$$

We shall show that $\mathcal{T}$ satisfies the conditions of Mönch's fixed point theorem. The proof will be given in the following four steps: 
Step 1. We show that $\mathcal{T}\left(\mathbb{B}_{R}\right) \subset \mathbb{B}_{R}$. From the hypothesis $\left(H_{2}\right)$ and Hölder's inequality, we have

$$
\begin{aligned}
& \left|(\mathcal{T} z)(t)(t-a)^{1-\gamma}\right| \\
& =\frac{1}{\Gamma(\gamma)} \frac{1}{(c+d-A)} \sum_{k=1}^{m} \frac{\lambda_{k}}{\Gamma(\mu)} \int_{a}^{\tau_{k}}\left(\tau_{k}-s\right)^{\mu-1}|f(s, z(s))| d s \\
& +\frac{1}{\Gamma(\gamma)}\left|\frac{d}{(c+d-A)}\right| \frac{1}{\Gamma(1-\gamma+\mu)} \int_{a}^{b}(b-s)^{-\gamma+\mu}|f(s, z(s))| d s \\
& +\frac{(t-a)^{1-\gamma}}{\Gamma(\mu)} \int_{a}^{t}(t-s)^{\mu-1}|f(s, z(s))| d s \\
& \leq \frac{1}{\Gamma(\gamma)} \frac{1}{(c+d-A)} \sum_{k=1}^{m} \frac{\lambda_{k}}{\Gamma(\mu)} \int_{a}^{\tau_{k}}\left(\tau_{k}-s\right)^{\mu-1}(s-a)^{\gamma-1} \rho(s)\|z\|_{C_{1-\gamma}} d s \\
& +\frac{1}{\Gamma(\gamma)}\left|\frac{d}{(c+d-A)}\right| \int_{a}^{b} \frac{(b-s)^{-\gamma+\mu}}{\Gamma(1-\gamma+\mu)}(s-a)^{\gamma-1} \rho(s)\|z\|_{C_{1-\gamma}} d s \\
& +\frac{(t-a)^{1-\gamma}}{\Gamma(\mu)} \int_{a}^{t}(t-s)^{\mu-1}(s-a)^{\gamma-1} \rho(s)\|z\|_{C_{1-\gamma}} d s \\
& \leq \frac{1}{\Gamma(\gamma)} \sum_{k=1}^{m} \frac{\lambda_{k}}{\Gamma(\mu)}\left(\int_{a}^{\tau_{k}} \frac{\left(\tau_{k}-s\right)^{(\mu-1) q}}{(c+d-A)}(s-a)^{(\gamma-1) q} d s\right)^{\frac{1}{q}}\|\rho\|_{L^{p}}\|z\|_{C_{1-\gamma}} \\
& +\frac{1}{\Gamma(\gamma)}\left|\frac{d}{(c+d-A)}\right|\left(\int_{a}^{b} \frac{(b-s)^{(-\gamma+\mu) q}}{\Gamma(1-\gamma+\mu)}(s-a)^{(\gamma-1) q} d s\right)^{\frac{1}{q}} \\
& \times\|\rho\|_{L^{p}}\|z\|_{C_{1-\gamma}}+\frac{(t-a)^{1-\gamma}}{\Gamma(\mu)} \\
& \times\left(\int_{a}^{t}(t-s)^{(\mu-1) q}(s-a)^{(\gamma-1) q} d s\right)^{\frac{1}{q}}\|\rho\|_{L^{p}}\|z\|_{C_{1-\gamma}} .
\end{aligned}
$$

Since $q>1, p>\frac{1}{\mu}$ and $\frac{1}{p}+\frac{1}{q}=1$, the change of variable $s=a-u\left(\tau_{k}-a\right)$ yields

$$
\left(\int_{a}^{\tau_{k}}\left(\tau_{k}-s\right)^{(\mu-1) q}(s-a)^{(\gamma-1) q} d s\right)^{\frac{1}{q}} \leq\left(\Lambda_{q, \mu, \gamma}\right)^{\frac{1}{q}}\left(\tau_{k}-a\right)^{\gamma+\mu-1}
$$

the change of variable $s=a-u(b-a)$ gives

$$
\left(\int_{a}^{b}(b-s)^{(-\gamma+\mu) q}(s-a)^{(\gamma-1) q} d s\right)^{\frac{1}{q}} \leq\left(\Delta_{q, \mu, \gamma}\right)^{\frac{1}{q}}(b-a)^{\mu},
$$

and the change of variable $s=a-u(t-a)$ gives us

$$
\left(\int_{a}^{t}(t-s)^{(\mu-1) q}(s-a)^{(\gamma-1) q} d s\right)^{\frac{1}{q}} \leq\left(\Lambda_{q, \mu, \gamma}\right)^{\frac{1}{q}}(t-a)^{\gamma+\mu-1} .
$$


Substitution of (3.10),(3.11) and (3.12) into (3.9) leads

$$
\begin{aligned}
& \left|(\mathcal{T} z)(t)(t-a)^{1-\gamma}\right| \\
\leq & \frac{1}{\Gamma(\gamma)} \frac{1}{(c+d-A)} \sum_{k=1}^{m} \frac{\lambda_{k}}{\Gamma(\mu)}\left(\Lambda_{q, \mu, \gamma}\right)^{\frac{1}{q}}\left(\tau_{k}-a\right)^{\gamma+\mu-1}\|\rho\|_{L^{p}}\|z\|_{C_{1-\gamma}} \\
& +\frac{1}{\Gamma(\gamma)}\left|\frac{d}{(c+d-A)}\right| \frac{1}{\Gamma(1-\gamma+\mu)}\left(\Delta_{q, \mu, \gamma}\right)^{\frac{1}{q}}(b-a)^{\mu}\|\rho\|_{L^{p}}\|z\|_{C_{1-\gamma}} \\
& +\frac{(t-a)^{1-\gamma}}{\Gamma(\mu)}\left(\Lambda_{q, \mu, \gamma}\right)^{\frac{1}{q}}(t-a)^{\gamma+\mu-1}\|\rho\|_{L^{p}}\|z\|_{C_{1-\gamma}} .
\end{aligned}
$$

For any $z \in \mathbb{B}_{R}$, we obtain

$$
\begin{aligned}
\|\mathcal{T} z\|_{C_{1-\gamma}} & \leq\left(\frac{1}{\Gamma(\gamma)} \frac{\left(\Lambda_{q, \mu, \gamma}\right)^{\frac{1}{q}}}{(c+d-A)} \sum_{k=1}^{m} \frac{\lambda_{k}}{\Gamma(\mu)}\left(\tau_{k}-a\right)^{\gamma+\mu-1}\right. \\
& \left.+\left(\frac{1}{\Gamma(\gamma)}\left|\frac{d}{(c+d-A)}\right| \frac{\left(\Delta_{q, \mu, \gamma}\right)^{\frac{1}{q}}}{\Gamma(1-\gamma+\mu)}+\frac{\left(\Lambda_{q, \mu, \gamma}\right)^{\frac{1}{q}}}{\Gamma(\mu)}\right)(b-a)^{\mu}\right)\|\rho\|_{L^{p}} R .
\end{aligned}
$$

By (H3), we have $\|\mathcal{T} z\|_{C_{1-\gamma}} \leq \mathcal{G} R \leq R$, that is, $\mathcal{T}\left(\mathbb{B}_{R}\right) \subset \mathbb{B}_{R}$.

Step 2. We shall prove that $\mathcal{T}$ is completely continuous.

The operator $\mathcal{T}$ is continuous. Let $\left\{z_{n}\right\}_{n \in \mathbb{N}}$ is a sequence such that $z_{n} \rightarrow z$ in $\mathbb{B}_{R}$.

Then for each $t \in J_{2}$, we have

$$
\begin{aligned}
& \left|\left(\left(\mathcal{T} z_{n}\right)(t)-(\mathcal{T} z)(t)\right)(t-a)^{1-\gamma}\right| \\
= & \frac{1}{\Gamma(\gamma)} \frac{1}{(c+d-A)} \sum_{k=1}^{m} \frac{\lambda_{k}}{\Gamma(\mu)} \int_{a}^{\tau_{k}}\left(\tau_{k}-s\right)^{\mu-1}\left|f\left(s, z_{n}(s)\right)-f(s, z(s))\right| d s \\
& +\frac{1}{\Gamma(\gamma)}\left|\frac{d}{(c+d-A)}\right| \int_{a}^{b} \frac{(b-s)^{-\gamma+\mu}}{\Gamma(1-\gamma+\mu)}\left|f\left(s, z_{n}(s)\right)-f(s, z(s))\right| d d s \\
& +\frac{(t-a)^{1-\gamma}}{\Gamma(\mu)} \int_{a}^{t}(t-s)^{\mu-1}\left|f\left(s, z_{n}(s)\right)-f(s, z(s))\right| d d s \\
\leq \quad & \frac{1}{\Gamma(\gamma)} \frac{1}{(c+d-A)} \sum_{k=1}^{m} \frac{\lambda_{k}}{\Gamma(\mu)} \int_{a}^{\tau_{k}}\left(\tau_{k}-s\right)^{\mu-1}(s-a)^{\gamma-1} d s \\
& \times\left\|f\left(\cdot, z_{n}(\cdot)\right)-f(\cdot, z(\cdot))\right\|_{C_{1-\gamma}} \\
& +\frac{1}{\Gamma(\gamma)}\left|\frac{d}{(c+d-A)}\right| \frac{1}{\Gamma(1-\gamma+\mu)} \int_{a}^{b}(b-s)^{-\gamma+\mu}(s-a)^{\gamma-1} d s \\
& \times\left\|f\left(\cdot, z_{n}(\cdot)\right)-f(\cdot, z(\cdot))\right\|_{C_{1-\gamma}} \\
& +\frac{(t-a)^{1-\gamma}}{\Gamma(\mu)} \int_{a}^{t}(t-s)^{\mu-1}(s-a)^{\gamma-1} d s\left\|f\left(\cdot, z_{n}(\cdot)\right)-f(\cdot, z(\cdot))\right\|_{C_{1-\gamma}} .
\end{aligned}
$$


Thus,

$$
\begin{aligned}
& \left|\left(\left(\mathcal{T} z_{n}\right)(t)-(\mathcal{T} z)(t)\right)(t-a)^{1-\gamma}\right| \\
\leq & \frac{1}{(c+d-A)} \frac{\mathcal{B}(\gamma, \mu)}{\Gamma(\mu) \Gamma(\gamma)} \sum_{k=1}^{m} \frac{\lambda_{k}\left(\tau_{k}-a\right)^{\gamma-1+\mu}}{\Gamma(\mu)}\left\|f\left(\cdot, z_{n}(\cdot)\right)-f(\cdot, z(\cdot))\right\|_{C_{1-\gamma}} \\
& +\left|\frac{d}{(c+d-A)}\right| \frac{(b-a)^{\mu}}{\Gamma(\mu+1)}\left\|f\left(\cdot, z_{n}(\cdot)\right)-f(\cdot, z(\cdot))\right\|_{C_{1-\gamma}} \\
& +\frac{(b-a)^{\mu}}{\Gamma(\mu)} \frac{\mathcal{B}(\gamma, \mu)}{\Gamma(\mu)}\left\|f\left(\cdot, z_{n}(\cdot)\right)-f(\cdot, z(\cdot))\right\|_{C_{1-\gamma}} .
\end{aligned}
$$

By (H1) and the Lebesgue dominated convergence theorem, we have

$$
\left\|\left(\mathcal{T} z_{n}-\mathcal{T} z\right)\right\|_{C_{1-\gamma}} \longrightarrow 0 \text { as } n \longrightarrow \infty
$$

which means that operator $\mathcal{T}$ is continuous on $\mathbb{B}_{R}$.

Step 3. $\mathcal{T}\left(\mathbb{B}_{R}\right)$ is relatively compact.

From Step 1, we have $\mathcal{T}\left(\mathbb{B}_{R}\right) \subset \mathbb{B}_{R}$. It follows that $\mathcal{T}\left(\mathbb{B}_{R}\right)$ is uniformly bounded i.e. $\mathcal{T}$ maps $\mathbb{B}_{R}$ into itself. Moreover, we show that operator $\mathcal{T}$ is equicontinuous on $\mathbb{B}_{R}$. Indeed, for any $a<t_{1}<t_{2}<b$ and $z \in \mathbb{B}_{R}$, we get

$$
\begin{aligned}
& \left|\left(t_{2}-a\right)^{1-\gamma}(\mathcal{T} z)\left(t_{2}\right)-\left(t_{1}-a\right)^{1-\gamma}(\mathcal{T} z)\left(t_{1}\right)\right| \\
\leq & \frac{1}{\Gamma(\mu)} \mid\left(t_{2}-a\right)^{1-\gamma} \int_{a}^{t_{2}}\left(t_{2}-s\right)^{\mu-1} f(s, z(s)) d s \\
& -\left(t_{1}-a\right)^{1-\gamma} \int_{a}^{t_{1}}\left(t_{1}-s\right)^{\mu-1} f(s, z(s)) d s \mid \\
\leq & \frac{\|f\|_{C_{1-\gamma}}}{\Gamma(\mu)} \mid\left(t_{2}-a\right)^{1-\gamma} \int_{a}^{t_{2}}\left(t_{2}-s\right)^{\mu-1}(s-a)^{\gamma-1} d s \\
& -\left(t_{1}-a\right)^{1-\gamma} \int_{a}^{t_{1}}\left(t_{1}-s\right)^{\mu-1}(s-a)^{\gamma-1} d s \mid \\
\leq \quad & \|f\|_{C_{1-\gamma}} \frac{\mathcal{B}(\gamma, \mu)}{\Gamma(\mu)}\left|\left(t_{2}-a\right)^{\mu}-\left(t_{1}-a\right)^{\mu}\right|,
\end{aligned}
$$

which tends to zero as $t_{2} \rightarrow t_{1}$, independent of $z \in \mathbb{B}_{R}$, where $\mathcal{B}(\cdot, \cdot)$ is a Beta function. Thus we conclude that $\mathcal{T}\left(\mathbb{B}_{R}\right)$ is equicontinuous on $\mathbb{B}_{r}$ and therefore is relatively compact. As a consequence of Steps 1 to 3 together with Arzela-Ascoli theorem, we conclude that $\mathcal{T}: \mathbb{B}_{R} \rightarrow \mathbb{B}_{R}$ is completely continuous operator.

Step 4. The Mönch condition is satisfied.

Let $\mathcal{V}$ be a subset of $\mathbb{B}_{R}$ such that $\mathcal{V} \subset \overline{c o}(\mathcal{T}(\mathcal{V}) \cup\{0\}) . \mathcal{V}$ is bounded and equicontinuous, and therefore the function $t \longrightarrow \alpha(\mathcal{V}(t))$ is continuous on $J_{1}$. By (H2)-(H3), 
Lemma 2.6, and the properties of the measure $\alpha$, for each $t \in J_{2}$

$$
\begin{aligned}
\alpha(\mathcal{V}(t)) \leq & \alpha(\mathcal{T}(\mathcal{V})(t) \cup\{0\}) \leq \alpha(\mathcal{T}(\mathcal{V})(t)) \\
\leq & \frac{1}{\Gamma(\gamma)} \frac{(t-a)^{\gamma-1}}{(c+d-A)} \sum_{k=1}^{m} \frac{\lambda_{k}}{\Gamma(\mu)} \int_{a}^{\tau_{k}}\left(\tau_{k}-s\right)^{\mu-1} \rho(s) \alpha(\mathcal{V}(s)) d s \\
& +\frac{1}{\Gamma(\gamma)}\left|\frac{d(t-a)^{\gamma-1}}{(c+d-A)}\right| \frac{1}{\Gamma(1-\gamma+\mu)} \int_{a}^{b}(b-s)^{-\gamma+\mu} \rho(s) \alpha(\mathcal{V}(s)) d s \\
& +\frac{1}{\Gamma(\mu)} \int_{a}^{t}(t-s)^{\mu-1} \rho(s) \alpha(\mathcal{V}(s)) d s \\
\leq & \frac{1}{\Gamma(\gamma)} \frac{(b-a)^{\gamma-1}}{(c+d-A)} \sum_{k=1}^{m} \frac{\lambda_{k}}{\Gamma(\mu)}\left(\int_{a}^{\tau_{k}}\left(\tau_{k}-s\right)^{(\mu-1) q} d s\right)^{\frac{1}{q}}\|\rho\|_{L^{p}} m \alpha(\mathcal{V}(b)) \\
& +\frac{1}{\Gamma(\gamma)}\left|\frac{d(b-a)^{\gamma-1}}{(c+d-A)}\right| \frac{1}{\Gamma(1-\gamma+\mu)}\left(\int_{a}^{b}(b-s)^{(-\gamma+\mu) q} d s\right)^{\frac{1}{q}} \\
& \times\|\rho\|_{L^{p}} \alpha(\mathcal{V}(b))+\frac{1}{\Gamma(\mu)}\left(\int_{a}^{t}(t-s)^{(\mu-1) q} d s\right)^{\frac{1}{q}}\|\rho\|_{L^{p}} \alpha(\mathcal{V}(b)) .
\end{aligned}
$$

From the facts

$$
\frac{1}{q}<1 \Longrightarrow \frac{1}{(\mu-1) q+1}<\frac{1}{\mu}, 1-\mu \neq \frac{1}{q},
$$

and

we get

$$
\frac{1}{q}<1 \Longrightarrow \frac{1}{(-\gamma+\mu) q+1}<\frac{1}{-\gamma+\mu+1}, \gamma-\mu \neq \frac{1}{q}
$$

$$
\begin{aligned}
\alpha(\mathcal{V}(t)) \leq & \left(\frac{m}{\Gamma(\gamma)} \frac{(b-a)^{\gamma-1}}{(c+d-A)} \sum_{k=1}^{m} \frac{\lambda_{k}\left(\tau_{k}-a\right)^{\mu}}{\Gamma(\mu+1)}\right. \\
& \left.+\frac{1}{\Gamma(\gamma)}\left|\frac{d}{(c+d-A)}\right| \frac{(b-a)^{\mu}}{\Gamma(-\gamma+\mu)}+\frac{(t-a)^{\mu}}{\Gamma(\mu+1)}\right)\|\rho\|_{L^{p}} \alpha(\mathcal{V}(b)) .
\end{aligned}
$$

It follows that

$$
\|\alpha(\mathcal{V})\|_{L^{\infty}}\left(1-L^{*}\right) \leq 0 .
$$

This means $\|\alpha(\mathcal{V})\|_{L^{\infty}}=0$, i.e. $\alpha(\mathcal{V}(t))=0$ for all $t \in J_{2}$. Thus $\mathcal{V}(t)$ is relatively compact in $E$. In view of Arzela-Ascoli theorem, $\mathcal{V}$ is relatively compact in $\mathbb{B}_{R}$. An application of Lemma 2.13 shows that $\mathcal{T}$ has a fixed point which is a solution of HNBVP (1.5)-(1.6).

Finally, we show that such a solution is indeed in $C_{1-\gamma}^{\gamma}\left(J_{1}, E\right)$. We apply $D_{a^{+}}^{\gamma}$ on both sides of (3.8), and using Lemmas 2.7, 2.8, to get

$$
D_{a^{+}}^{\gamma} z(t)=D_{a^{+}}^{\gamma} I_{a^{+}}^{\mu} f(t, z(t))=D_{a^{+}}^{\nu(1-\mu)} f(t, z(t)) .
$$

Since $f(\cdot, z(\cdot)) \in C_{1-\gamma}^{\nu(1-\mu)}\left(J_{1}, E\right)$, it follows by definition of the space $C_{1-\gamma}^{\nu(1-\mu)}\left(J_{1}, E\right)$ that $D_{a^{+}}^{\gamma} z(t) \in C_{1-\gamma}\left(J_{1}, E\right)$ which implies that $z(t) \in C_{1-\gamma}^{\gamma}\left(J_{1}, E\right)$. The proof is complete. 


\section{An example}

We consider the Hilfer fractional differential equation with nonlocal boundary condition

$$
\left\{\begin{array}{l}
D_{0^{+}}^{\mu, \nu} z(t)=f(t, z(t)), \quad t \in(0,1], \quad 0<\mu<1,0 \leq \nu \leq 1 \\
I_{0^{+}}^{1-\gamma}\left[\frac{1}{4} z\left(0^{+}\right)+\frac{3}{4} z\left(1^{-}\right)\right]=\frac{2}{5} z\left(\frac{2}{3}\right), \quad \mu \leq \gamma=\mu+\nu(1-\mu)
\end{array}\right.
$$

where $f(t, z(t))=\frac{1}{16} t \sin |z(t)|, \mu=\frac{1}{3}, \nu=\frac{1}{4}, \gamma=\frac{1}{2}, c=\frac{1}{4}, d=\frac{3}{4}, \lambda_{1}=\frac{2}{5}$ and $m=1, \tau_{1}=\frac{2}{3}$. Let $E=\mathbb{R}^{+}$and $J_{2}=(0,1]$.

Clearly we can see that

$$
\sqrt{t} f(t, z)=\frac{1}{16} \sqrt[3]{t} \sin z \in C\left([0,1], \mathbb{R}^{+}\right),
$$

and hence $f(t, z) \in C_{\frac{1}{2}}\left([0,1], \mathbb{R}^{+}\right)$. Also, observe that, for $t \in(0,1]$ and for any $z \in C_{\frac{1}{2}}\left([0,1], \mathbb{R}^{+}\right)$,

$$
\|f(t, z)\| \leq \frac{1}{16} t\|z\|
$$

Therefore, the conditions (H1) and (H2) is satisfied with $\rho(t)=\frac{1}{16} t \in L^{p}(0,1)$. Select $p=4$, we have

$$
\|\rho\|_{L^{4}}=\left(\int_{0}^{1}\left|\frac{1}{16} s\right|^{4} d s\right)^{\frac{1}{4}}=\frac{327680^{\frac{3}{4}}}{327680} .
$$

It is easy to check that conditions in (H3) are satisfied too. Indeed, by some simple computations with $q=\frac{4}{3}$, we get

$$
\Lambda_{q, \mu, \gamma}=\frac{\Gamma(q(\mu-1)+1) \Gamma(q(\gamma-1)+1)}{\Gamma(q(\mu+\gamma-2)+2)}=\frac{\Gamma\left(\frac{1}{9}\right) \Gamma\left(\frac{1}{3}\right)}{\Gamma\left(\frac{4}{9}\right)},
$$

and

$$
\Delta_{q, \mu, \gamma}=\frac{\Gamma(q(\mu-\gamma)+1) \Gamma(q(\gamma-1)+1)}{\Gamma(q(\mu-1)+2)}=\frac{\Gamma\left(\frac{7}{9}\right) \Gamma\left(\frac{1}{3}\right)}{\Gamma\left(\frac{10}{9}\right)},
$$

also, we have

$$
A=\lambda_{1} \frac{\left(\tau_{1}\right)^{\gamma-1}}{\Gamma(\gamma)}=\frac{\sqrt{\frac{6}{\pi}}}{5}
$$

It follows that $\mathcal{G} \simeq 0.35<1$, and $L^{*} \simeq 0.06<1,(m=1)$. An application of Theorem 3.3 implies that problem $(4.1)$ has a solution in $C_{\frac{1}{2}}^{\frac{1}{2}}\left([0,1], \mathbb{R}^{+}\right)$.

Acknowledgements. The authors would like to thank the referees for their careful reading of the manuscript and insightful comments, which helped improve the quality of the paper. The authors would also like to acknowledge the valuable comments and suggestions from the editors, which vastly contributed to the improvement of the presentation of the paper. 


\section{References}

[1] Abbas, S., Benchohra, M., Lazreg, J.E., Zhou, Y., A survey on Hadamard and Hilfer fractional differential equations: analysis and stability, Chaos Solitons Fractals, 102(2017), 47-71.

[2] Abdo, M.S., Panchal, S.K., Fractional integro-differential equations involving $\psi$-Hilfer fractional derivative, Adv. Appl. Math. Mech., 11(2019), no. 2, 338-359.

[3] Abdo, M.S., Panchal, S.K., Saeed, A.M., Fractional Boundary value problem with $\psi$-Caputo fractional derivative, Proc. Indian Acad. Sci. (Math. Sci.), 129(2019), no. 5,65 .

[4] Agarwal, R.P., Benchohra, M., Hamani, S., A survey on existence results for boundary value problems of nonlinear fractional differential equations and inclusions, Acta Appl. Math., 109(2010), no. 3, 973-1033.

[5] Bhairat, S.P., Existence and continuation of solution of Hilfer fractional differential equations, J. Math. Model., 7(2018), no. 1, 1-20.

[6] Bhairat, S.P., Dhaigude, D.B., Local existence and uniqueness of solutions for HilferHadamard fractional differential problem, Nonlinear Dyn. Syst. Theory, 18(2018), no. 2, 144-153.

[7] Bhairat, S.P., Dhaigude, D.B., Existence of solutions of generalized fractional differential equation with with nonlocal initial condition, Math. Bohem., 144(2019), no. 2, 203-220.

[8] Dhaigude, D.B., Bhairat, S.P., Existence and uniqueness of solution of Cauchy-type problem for Hilfer fractional differential equations, Commun. Appl. Anal., 22(2018), no. $1,121-134$.

[9] Diethelm, K., The analysis of fractional differential equations, J. Math. Anal. Appl., 265(2004), 229-248.

[10] Furati, K.M., Kassim, M.D., Existence and uniqueness for a problem involving Hilfer fractional derivative, Comput. Math. Appl., 64(2012), no. 6, 1616-1626.

[11] Furati, K.M., Tatar, N.E., An existence result for a nonlocal fractional differential problem, J. Fract. Calc. Appl., 26(2004), 43-54.

[12] Gaafar, F.M., Continuous and integrable solutions of a nonlinear Cauchy problem of fractional order with nonlocal coditions, J. Egyptian Math. Soc., 22(2014), no. 3, 341347.

[13] Gonzalez, C., Melado, A.J., Fuster, E.L., A Monch type fixed point theorem under the interior condition, J. Math. Anal. Appl., 352(2009), no. 2, 816-821.

[14] Granas, A., Dugundji, J., Fixed Point Theory, Springer Monographs in Mathematics, Springer-Verlag, New York, 2003.

[15] Hilfer, R., Applications of Fractional Calculus in Physics, World Scientific, Singapore, 2000 .

[16] Hilfer, R., Luchko, Y., Tomovski, Z., Operational method for the solution of fractional differential equations with generalized Riemann-Lioville fractional derivative, Fract. Calc. Appl. Anal., 12(2009), no. 3, 289-318.

[17] Kilbas, A.A., Srivastava, H.M., Trujillo, J.J., Theory and Applications of Fractional Differential Equations, North-Holland Math. Stud., 204 Elsevier, Amsterdam 2006.

[18] Mönch, H., Boundary value problem for nonlinear ordinary differential equations of second order in Banach spaces, Nonlinear Anal., 75(1980) no. 5, 985-999. 
[19] Sabri, T.M., Ahmed, B., Agarwal, R.P., On abstract Hilfer fractional integrodifferential equations with boundary conditions, Arab J. Math. Sci., (2019).

[20] Vivek, D., Kanagarajan, K., Elsayed, E.M., Some existence and stability results for Hilfer-fractional implicit differential equations with nonlocal conditions, Mediterr. J. Math., 15(2018), no. 1, 1-15.

[21] Szufla, S., On the application of measure of noncompactness to existence theorems, Rend. Semin. Mat. Univ. Padova, 75(1986), 1-14.

[22] Wang, J., Zhang, Y., Nonlocal initial value problems for differential equations with Hilfer fractional derivative, Appl. Math. Comput., 266(2015), 850-859.

Hanan A. Wahash

Department of Mathematics,

Dr. Babasaheb Ambedkar Marathwada University,

Aurangabad - 431001, (M.S) India

e-mail: hawahash86@gmail.com

Mohammed S. Abdo

Department of Mathematics,

Dr. Babasaheb Ambedkar Marathwada University,

Aurangabad - 431001, (M.S) India,

Hodeidah University, Al-Hodeidah 31141, Yemen

e-mail: msabdo1977@gmail.com

Satish K. Panchal

Department of Mathematics,

Dr. Babasaheb Ambedkar Marathwada University,

Aurangabad - 431001, (M.S) India

e-mail: drpanchalsk@gmail.com

Sandeep P. Bhairat

Faculty of Engineering Mathematics,

Institute of Chemical Technology Mumbai,

Marathwada Campus, Jalna - 431203 (M.S) India

(Corresponding author)

e-mail: sp.bhairat@marj.ictmumbai.edu.in 